\title{
Exploration of shared genetic susceptibility loci between type 1 diabetes and rheumatoid arthritis in the Pakistani population
}

\author{
Muhammad Muaaz Aslam ${ }^{1,2}$, Peter John', Kang-Hsien Fan², Attya Bhatti ${ }^{1}$, Sidrah Jahangir ${ }^{1}$, Eleanor Feingold ${ }^{2}$, \\ F. Yesim Demirci ${ }^{2}$ and M. Ilyas Kamboh ${ }^{2^{*}}$
}

\begin{abstract}
Objective: Type 1 diabetes (T1D) and rheumatoid arthritis (RA) are autoimmune diseases. It is known that certain genetic loci and factors that increase the overall autoimmunity risk can be shared among different autoimmune diseases. We sought to replicate seven T1D-related SNPs (single nucleotide polymorphisms) that have been previously reported to be associated with RA susceptibility in a small set of mixed family-based and case-control Pakistani sample in a relatively large and independent RA case-control sample from the same population. Seven T1D-associated SNPs (GLIS3/rs7020673, BACH2/rs1 1755527, SKAP2/rs7804356, GDSMB/rs2290400, C6orf173/rs9388489, LOC399716/ rs947474 and DLK1-MEG2/rs941576) were genotyped in a large Pakistani RA case-control sample $(n=1959)$ using TaqMan ${ }^{\circledR}$ SNP genotyping assays.

Results: None of the tested SNPs showed statistically significant association with RA susceptibility; however, one SNP (GLIS3/rs7020673) showed a trend for association ( $O R=0.88, \mathrm{p}=7.99 \mathrm{E}-02)$. Our study has failed to replicate the previously reported association of seven T1D-associated SNPs with RA risk in a large sample from the same population. Thus, our results do not support a major role of these T1D SNPs in affecting RA susceptibility in the Pakistani population.
\end{abstract}

Keywords: Type 1 diabetes, Rheumatoid arthritis, Association, Shared genetic

\section{Introduction}

Prevalence of autoimmune diseases is $3-5 \%$ in the general population [1,2]. Observation of poly-autoimmunity (more than one autoimmune disease in the same person) and/or familial autoimmunity (more than one autoimmune disease in the same family) have suggested that autoimmune diseases share some genetic risk factors and biological pathways [3]. Consistent with this observation, some genetic loci/genes have been found to be associated with multiple autoimmune diseases such as $6 \mathrm{p} 21 / M H C$, 2q33/CTLA4, 2q32/STAT4, 18p11/PTPN2, 1q32/IL10, 7q32/IRF5, 1p31/IL23R [4]. These shared loci/genes may influence the susceptibility for different diseases through

\footnotetext{
*Correspondence: kamboh@pitt.edu

2 Department of Human Genetics, Graduate School of Public Health

(GSPH), University of Pittsburgh, Pittsburgh, PA 15216, USA

Full list of author information is available at the end of the article
}

the effects of the same or different genetic variations [5-7].

Rheumatoid arthritis (RA) is a complex, systemic, chronic, inflammatory, autoimmune disease characterized by synovial inflammation of mainly small joints and autoantibodies production, which may lead to physical disability associated with the socioeconomic burden and in severe cases, an early death $[8,9]$. The interplay of genetic and environmental factors triggers the onset of RA with an estimated heritability of $\sim 60 \%$ [10]. Multiple genome-wide association studies (GWAS), have identified $\sim 50$ genetic loci for RA susceptibility [11], with a major contribution from the MHC locus [12].

Type1 diabetes (T1D) is an autoimmune disease characterized by hyperglycemia due to the attack of body's own immune system on $\beta$-cells in the pancreatic islets, reducing the production of insulin which may lead to major health issues such as heart disease, kidney 
failure, ketoacidosis, blindness and stroke [13, 14]. Contrary to many other autoimmune diseases, which predominantly affect women, T1D affects both genders with a moderately higher prevalence in males at a relatively young age [15]. According to epidemiological data, the incidence of T1D varies globally [16]. To date more than 40 genetic loci have been found to be associated with T1D risk [17].

The concurrence of T1D and RA have been reported within the same individuals and families; however, the shared genetic factors between these two conditions are not fully identified. $H L A-D R B 1$ in the MHC class II region is a major shared genetic locus between these two diseases $[18,19]$. Among non-MHC loci, PTPN22, AFF3, CTLA4, TNFAIP3 and TAGAP have been reported as shared risk loci between T1D and RA [20-22]. A previous study conducted in a small set of RA Pakistani sample reported seven T1D-implicated risk variants to be associated with RA risk [23]. In order to replicate or refute this reported association, the present study was conducted in a much larger Pakistani RA case-control sample on the same seven T1D-implicated risk variants.

\section{Main text \\ Materials and methods \\ Study subjects}

A total of 1959 unrelated subjects (1222 cases, 737 controls) were recruited for this study. Blood samples and relevant clinical information were collected from two public (Military Hospital and Pakistani Institute of Medical Sciences) and one private (Rehmat Noor Clinic) rheumatology center in Pakistan. All cases recruited in this study (mean age $\pm \mathrm{SD}=43.1 \pm 12.33,78 \%$ women) met the 1987 ACR (American College of Rheumatology) classification criteria for RA [24]. The control subjects (mean age $\pm \mathrm{SD}=40.7 \pm 12.49,39.5 \%$ women) had no history of autoimmune diseases at the time of enrollment. A screening questionnaire was filled out and an informed consent obtained from each subject at the time of the recruitment. All blood samples were collected in EDTA tubes and processed shortly after the collection. The study was approved by the Institutional review board (IRB) of the University of Pittsburgh, USA (IRB no. PRO12110472).

\section{Genomic DNA extraction}

DNA was extracted from whole blood shortly after collection using either standard phenol-chloroform extraction method or GeneJET Whole Blood Genomic DNA Purification kit (Thermo Scientific USA) and quantified using NanoDrop ${ }^{\text {TM }} 2000$ spectrophotometer (Thermo Scientific USA).

\section{SNP selection}

Seven T1D-associated SNPs which were previously reported to be significantly $(\mathrm{p}<0.05)$ associated with RA in a relatively small Pakistani study sample [23] were selected for genotyping. All the relevant information on the selected SNPs is provided in Table 1.

\section{Genotyping}

Genotyping of selected SNPs was performed by using available (functionally tested) TaqMan ${ }^{\circledR}$ assays (Applied Biosystems, ThermoFisher Scientific) following the manufacturer's instructions. After thermal cycling of the TaqMan assays and DNA on 384-well plates, the endpoint fluorescence reading was performed on a QuantStudio $^{\mathrm{TM}} 12 \mathrm{~K}$ Flex system (Applied Biosystems, ThermoFisher Scientific). To test the genotyping consistency, $18 \%$ of samples were included as the replicates in genotyping runs.

\section{Statistical analysis}

Minor allele frequency (MAF) for each genotype was calculated based on non-missing allele counts. Congruity to Hardy-Weinberg Equilibrium (HWE) was tested using Chi square goodness of fit test. $\mathrm{p}<1 \mathrm{E}-05$ was used to define a significant deviation from HWE as part of the quality control for genotyped SNPs. Logistic regression analysis was performed under the additive model for association analysis of SNPs with RA risk using age and sex as covariates. $\mathrm{p}<0.05$ was considered as suggestive evidence of association and Benjamin Hochberg false discovery rate (FDR) (q-value) of $<0.20$ was considered as statistically significant similarly done in previous studies $[25,26]$. All analyses were implemented in R, version 3.4.4. Power analysis was performed using the Quanto Program (http://biostats.usc.edu/Quanto.html).

\section{Results}

A total of 1959 unrelated RA case-control subjects were genotyped to replicate the previously reported association (by Kiani et al. [23]) of seven T1D-associated SNPs with RA. The genotype call rates were more than 90\% for all SNPs and the genotype distribution did not deviate significantly from HWE for any of them. All tested SNPs showed similar frequencies in our study as reported by Kiani et al. [23]. Of the seven tested SNPs, only one (rs947474) was an upstream gene variant and the rest were intronic. None of the tested T1D-associated SNPs showed significant association with RA in our large replication sample. However, a trend for association $(\mathrm{OR}=0.88 ; \mathrm{p}=7.99 \mathrm{E}-02, \mathrm{FDR}=2.93 \mathrm{E}-01)$ was observed for one intronic SNP (rs7020673) located in the GLIS3 gene (Table 1). For the seven SNPs tested in 


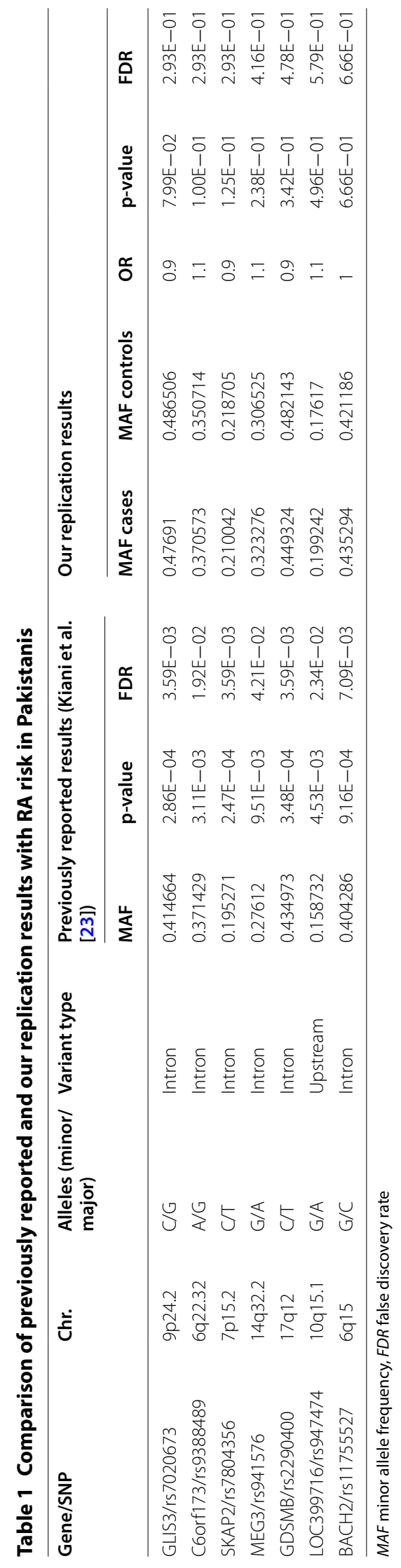


this study with MAFs ranging between 0.16 and 0.43 , we had $>80 \%$ power to detect significant association at an effect size of $\geq 1.3$ under the additive model.

\section{Discussion}

Seven T1D-associated SNPs were previously reported to be associated with RA susceptibility in the Pakistani population using a mixed family-based and unrelated RA case-control sample of 366 subjects [23]. In our study, we genotyped those seven SNPs in a much larger Pakistani sample (1959 unrelated RA cases and controls) and found no significant association with RA susceptibility. However, one SNP (GLIS3/rs7020673) showed a trend for the association. This SNP was strongly associated $(\mathrm{p}=5.29 \mathrm{E}-05)$ with T1D susceptibility in the Pakistani population [27]. In addition to T1D, polymorphisms in GLIS3 have been reported to be associated with type 2 diabetes, Alzheimer's disease and osteoarthritis [28, 29]. It is possible that genetic variation in GLIS3 may also have some role in the RA etiology, but this awaits confirmation in much larger genetic studies.

The difference between the previous Pakistani study [23] and the current study could be related to the differences in the study design and power. The analysis of the previous study was done on a smaller set of combined family-based and unrelated RA cases and controls and that may have inflated the significance level. Our study was sufficiently powered, as we had $>80 \%$ power to detect significant associations at an effect size of $\geq 1.3$ ( $>70 \%$ power at an effect size of $\geq 1.25$ ) under the additive model. Alternatively, it is possible that some of those SNPs may be more relevant to the development of familial RA than the sporadic RA since the previous study used a combination of case-control and familial subjects analyzed by FamCC (Family Case Control) software [30].

\section{Conclusion}

In conclusion, our study on a larger RA case-control sample has failed to replicate the previously reported seven T1D-implicated SNPs with RA risk in Pakistanis. The difference in study design, power and contrasting statistical models for data analysis may explain the different outcomes.

\section{Limitations}

Our study could not replicate the previous reported association of T1D-related SNPs with RA in Pakistanis. However, the absence of association should be interpreted cautiously, because the present study is limited by its sample size and sample type. As we specifically explored Pakistani population, the results from this study should not be generalized to other populations. We only tested seven T1D associated SNPs and there is a need to test other T1D-implicated SNPs to explore the shared genetic of RA and T1D.

\begin{abstract}
Abbreviations
T1D: type 1 diabetes; RA: rheumatoid arthritis; GWAS: genome-wide association studies; ACR: American College of Rheumatology; IRB: Institutional Review Board; MAF: minor allele frequency; HWE: Hardy-Weinberg Equilibrium; FDR: false discovery rate; FamCC: family case control; SNPs: single nucleotide polymorphisms.
\end{abstract}

\section{Acknowledgements}

We acknowledge the Higher Education Commission (HEC) for supporting this study within Pakistan and the University of Pittsburgh for providing facilities to support this study in the USA. We are grateful to all Rheumatologist for referring patients for recruitment and nursing staff for collecting blood samples. We are highly thankful to all subjects for participating in this study.

\section{Authors' contributions}

MMA: Study design, genotyping, interpretation, manuscript preparation; PJ: Sample preparation; KF: Data analysis; AB: Clinical data interpretation; SJ: Sample collection, sample processing; EF: Data interpretation; FYD: Genotyping, quality control; MIK: Study design, manuscript preparation. All authors read and approved the final manuscript.

\section{Funding}

None.

\section{Availability of data}

The data used to support the findings of this study are included within the article.

Ethics approval and consent to participate

The present study was approved by the IRB of University of Pittsburgh, Pittsburgh, PA, USA (IRB No. PRO12110472). Written inform consent was obtained from all the study participants prior to their inclusion in this study.

\section{Consent for publication}

Not applicable.

\section{Competing interests}

The authors declare that they have no competing interests.

\section{Author details}

${ }^{1}$ Atta-ur-Rahman School of Applied Biosciences (ASAB), National University of Sciences and Technology (NUST), Islamabad, Pakistan. ${ }^{2}$ Department of Human Genetics, Graduate School of Public Health (GSPH), University of Pittsburgh, Pittsburgh, PA 15216, USA.

Received: 24 July 2019 Accepted: 21 August 2019

Published online: 27 August 2019

\section{References}

1. Eaton WW, Rose NR, Kalaydjian A, Pedersen MG, Mortensen PB. Epidemiology of autoimmune diseases in Denmark. J Autoimmun. 2007:29(1):1-9.

2. Hayter SM, Cook MC. Updated assessment of the prevalence, spectrum and case definition of autoimmune disease. Autoimmun Rev. 2012;11(10):754-65.

3. Anaya J-M. Common mechanisms of autoimmune diseases (the autoimmune tautology). Autoimmun Rev. 2012;11(11):781-4.

4. Cotsapas C, Voight BF, Rossin E, Lage K, Neale BM, Wallace C, et al. Pervasive sharing of genetic effects in autoimmune disease. PLoS Genet. 2011;7(8):e1002254

5. Smyth DJ, Plagnol V, Walker NM, Cooper JD, Downes K, Yang JHM, et al. Shared and distinct genetic variants in type 1 diabetes and celiac disease. N Engl J Med. 2008;359(26):2767-77. 
6. Sirota M, Schaub MA, Batzoglou S, Robinson WH, Butte AJ. Autoimmune disease classification by inverse association with SNP alleles. PLoS Genet. 2009:5(12):e1000792.

7. Ramos PS, Criswell LA, Moser KL, Comeau ME, Williams AH, Pajewski NM, et al. A comprehensive analysis of shared loci between systemic lupus erythematosus (SLE) and sixteen autoimmune diseases reveals limited genetic overlap. PLoS Genet. 2011;7(12):e1002406.

8. McInnes IB, Schett G. The pathogenesis of rheumatoid arthritis. N Engl J Med. 2011:365(23):2205-19.

9. Aslam MM, John P, Bhatti A, Jahangir S, Kamboh MI. Vitamin D as a principal factor in mediating rheumatoid arthritis-derived immune response. Biomed Res Int. 2019;2019:1-12.

10. de Vries R. Genetics of rheumatoid arthritis: time for a change! Curr Opin Rheumatol. 2011:23(3):227-32.

11. Ito Y, Sakaguchi S. Rheumatoid arthritis, genetics BT-encyclopedia of medical immunology: autoimmune diseases. New York: Springer; 2014. p. 996-1001.

12. van der Woude D, Houwing-Duistermaat JJ, Toes RE, Huizinga TW, Thomson W, Worthington J, et al. Quantitative heritability of anti-citrullinated protein antibody-positive and anti-citrullinated protein antibody-negative rheumatoid arthritis. Arthritis Rheum. 2009;60(4):916-23.

13. Atkinson MA, Eisenbarth GS. Type 1 diabetes: new perspectives on disease pathogenesis and treatment. Lancet (London, England). 2001;358(9277):221-9.

14. Van Belle TL, Coppieters KT, Von Herrath MG. Type 1 diabetes: etiology, immunology, and therapeutic strategies. Physiol Rev. 2011;91(1):79-118.

15. Atkinson MA, Eisenbarth GS, Michels AW. Type 1 diabetes. Lancet. 2014;383(9911):69-82.

16. Maahs DM, West NA, Lawrence JM, Mayer-Davis EJ. Epidemiology of type 1 diabetes. Endocrinol Metab Clin North Am. 2010;39(3):481-97.

17. Concannon P, Rich SS, Nepom GT. Genetics of type 1A diabetes. N Engl J Med. 2009;360(16):1646-54.

18. Noble JA, Valdes AM. Genetics of the HLA region in the prediction of type 1 diabetes. Curr Diab Rep. 2011;11(6):533-42.

19. Holoshitz J. The rheumatoid arthritis HLA-DRB1 shared epitope. Curr Opin Rheumatol. 2010;22(3):293-8.

20. Chelala C, Duchatelet S, Joffret ML, Bergholdt R, Dubois-Laforgue D, Ghandil P, et al. PTPN22 R620W functional variant in type 1 diabetes and autoimmunity related traits. Diabetes. 2007;56(2):522-6.
21. Barton A, Eyre S, Ke X, Hinks A, Bowes J, Flynn E, et al. Identification of AF4/FMR2 family, member 3 (AFF3) as a novel rheumatoid arthritis susceptibility locus and confirmation of two further pan-autoimmune susceptibility genes. Hum Mol Genet. 2009;18(13):2518-22

22. Eyre S, Hinks A, Bowes J, Flynn E, Martin P, Wilson AG, et al. Overlapping genetic susceptibility variants between three autoimmune disorders: rheumatoid arthritis, type 1 diabetes and coeliac disease. Arthritis Res Ther. 2010;12(5):R175

23. Kiani AK, Jahngir S, John P, Bhatti A, Zia A, Wang X, et al. Genetic link of type 1 diabetes susceptibility loci with rheumatoid arthritis in Pakistani patients. Immunogenetics. 2015;67(5):277-82.

24. Arnett FC, Edworthy SM, Bloch DA, McShane DJ, Fries JF, Cooper NS, et al. The American Rheumatism Association 1987 revised criteria for the classification of rheumatoid arthritis. Arthritis Rheum. 1988;31(3):315-24.

25. Radwan ZH, Wang X, Waqar F, Pirim D, Niemsiri V, Hokanson JE, et al. Comprehensive evaluation of the association of APOE genetic variation with plasma lipoprotein traits in U.S. whites and African blacks. PLOS ONE. 2014;9(12):e114618

26. Niemsiri V, Wang X, Pirim D, Radwan ZH, Bunker CH, Barmada MM, et al. Genetic contribution of SCARB1 variants to lipid traits in African Blacks: a candidate gene association study. BMC Med Genet. 2015;16:106.

27. Kiani AK, John P, Bhatti A, Zia A, Shahid G, Akhtar P, et al. Association of 32 type 1 diabetes risk loci in Pakistani patients. Diabetes Res Clin Pract. 2015:108(1):137-42.

28. Calderari S, Ria M, Gérard C, Nogueira TC, Villate O, Collins SC, et al. Molecular genetics of the transcription factor GLIS3 identifies its dual function in beta cells and neurons. Genomics. 2018:110(2):98-111.

29. Casalone E, Tachmazidou I, Zengini E, Hatzikotoulas K, Hackinger S, Suveges $\mathrm{D}$, et al. A novel variant inGLIS3is associated with osteoarthritis. Ann Rheum Dis. 2018;77(4):620-3.

30. Zhu X, Li S, Cooper RS, Elston RC. A Unified association analysis approach for family and unrelated samples correcting for stratification. Am J Hum Genet. 2008:82(2):352-65.

\section{Publisher's Note}

Springer Nature remains neutral with regard to jurisdictional claims in published maps and institutional affiliations.
Ready to submit your research? Choose BMC and benefit from:

- fast, convenient online submission

- thorough peer review by experienced researchers in your field

- rapid publication on acceptance

- support for research data, including large and complex data types

- gold Open Access which fosters wider collaboration and increased citations

- maximum visibility for your research: over 100M website views per year

At BMC, research is always in progress.

Learn more biomedcentral.com/submissions 